\title{
Common fixed point theorems for two hybrid pairs of mappings satisfying the common property (E.A) in Menger PM-spaces
}

\section{Zhaoqi Wu', Chuanxi Zhu' ${ }^{1 *}$ and Jin $\mathrm{Li}^{2}$}

"Correspondence:

chuanxizhu@126.com

${ }^{1}$ Department of Mathematics,

Nanchang University, Nanchang,

330031, P.R. China

Full list of author information is

available at the end of the article

\begin{abstract}
In this paper, a new concept of the common property (E.A) for two hybrid pairs of mappings is introduced in Menger PM-spaces. Utilizing this concept, some common fixed point theorems, which shed some new light on the study of fixed point results for hybrid pairs in Menger PM-spaces, are obtained under strict contractive conditions. The corresponding results in metric spaces which generalize many known results are also obtained. Finally, an example is also given to exemplify our main results.
\end{abstract}

Keywords: common fixed point; Menger PM-space; hybrid pair; common property (E.A)

\section{Introduction}

The concept of a probabilistic metric space was initiated and studied by Menger which is a generalization of the metric space notion $[1,2]$. The theory of a probabilistic metric space is an active field and has applications in many other branches of mathematics such as cluster analysis, mathematical statistics and chaos theory $[3,4]$. It has also been applied to quantum particle physics in connection with both string and $\epsilon^{\infty}$ theory [5].

Fixed point theory in a probabilistic metric space is an important branch of probabilistic analysis, which is closely related to the existence and uniqueness of solutions of differential equations and integral equations [6, 7]. Many results on the existence of fixed points or solutions of nonlinear equations under various types of conditions in Menger spaces have been extensively studied by many scholars (see, e.g., $[8,9]$ ).

Jungck [10] introduced the concept of compatible mappings in metric spaces and proved some common fixed point theorems. In [11], the concept of weakly compatible mappings was given. The concept of compatible mappings in a Menger space was initiated by Mishra [12], and since then many fixed point results for compatible mappings and weakly compatible mappings have been studied [13-16]. The study for noncompatible mappings is also interesting. This was initiated and studied by Pant first in metric spaces [17-20]. In 2002, Aamri and Moutawakil defined a new property for a pair of mappings, i.e., the so-called property (E.A), which is a generalization of noncompatibility [21]. Using this property, some common fixed point theorems under strict contractive conditions in metric spaces have been given. In 2004, Kamran introduced the concept of the property (E.A) in a hybrid case in metric spaces and obtained some coincidence and fixed points theo-

(c) 2013 Wu et al: licensee Springer. This is an Open Access article distributed under the terms of the Creative Commons Attribution License (http://creativecommons.org/licenses/by/2.0), which permits unrestricted use, distribution, and reproduction in any medium, provided the original work is properly cited. 
rems for hybrid strict contractions [22]. However, Sintunavarat and Kumam pointed out that one condition in one of their main results is superfluous [23]. Liu et al. defined the concept of the common property (E.A) for single-valued as well as hybrid pairs of mappings in metric spaces and obtained many interesting results [24]. Utilizing these concepts, many authors studied the existence of coincidence and fixed points in symmetric spaces [25-28].

On the other hand, fixed point results for mappings under strict contractive conditions in probabilistic metric spaces are not very fruitful. In 2009, Fang defined the property (E.A) for two single-valued mappings in Menger PM-spaces and studied the existence of common fixed points in such spaces [29]. In 2011, Ali et al. obtained some common fixed point results for strict contractions in Menger PM-spaces using the common property (E.A) for two pairs of single-valued mappings [30].

The purpose of this paper is to introduce the concept of the common property (E.A) for two hybrid pairs of mappings in Menger PM-spaces and study the existence of coincidence and common fixed points for pairs of mappings satisfying such a property under strict contractive conditions. We also obtain some corresponding results under strict contractive conditions in metric spaces.

\section{Preliminaries}

A mapping $F: \mathbb{R} \rightarrow \mathbb{R}^{+}$is called a distribution function if it is nondecreasing leftcontinuous with $\sup _{t \in \mathbb{R}} F(t)=1$ and $\inf _{t \in \mathbb{R}} F(t)=0$.

We will denote by $\mathscr{D}$ the set of all distribution functions, while $H$ will always denote the specific distribution function defined by

$$
H(t)= \begin{cases}0, & t \leq 0, \\ 1, & t>0 .\end{cases}
$$

Let $F_{1}, F_{2} \in \mathscr{D}$. The algebraic sum $F_{1} \oplus F_{2}$ is defined by

$$
\left(F_{1} \oplus F_{2}\right)(t)=\sup _{t_{1}+t_{2}=t} \min \left\{F_{1}\left(t_{1}\right), F_{2}\left(t_{2}\right)\right\}
$$

for all $t \in \mathbb{R}$.

Let $f$ and $g$ be two functions defined on $\mathbb{R}$ with positive values. The notation $f>g$ means that $f(t) \geq g(t)$ for all $t \in \mathbb{R}$, and there exists at least one $t_{0} \in \mathbb{R}$ such that $f\left(t_{0}\right)>g\left(t_{0}\right)$.

A mapping $\Delta:[0,1] \times[0,1] \rightarrow[0,1]$ is called a triangular norm (for short, a $t$-norm) if the following conditions are satisfied:

(1) $\Delta(a, 1)=a$;

(2) $\Delta(a, b)=\Delta(b, a)$;

(3) $\Delta(a, c) \geq \Delta(b, d)$ for $a \geq b, c \geq d$;

(4) $\Delta(a, \Delta(b, c))=\Delta(\Delta(a, b), c)$.

Definition 2.1 [4] A triplet $(X, \mathcal{F}, \Delta)$ is called a Menger probabilistic metric space (for short, a Menger PM-space) if $X$ is a nonempty set, $\Delta$ is a $t$-norm and $\mathcal{F}$ is a mapping from $X \times X$ into $\mathscr{D}$ satisfying the following conditions (we denote $\mathcal{F}(x, y)$ by $F_{x, y}$ ):

(MS-1) $F_{x, y}(t)=H(t)$ for all $t \in R$ if and only if $x=y$;

(MS-2) $F_{x, y}(t)=F_{y, x}(t)$ for all $t \in R$; 
(MS-3) $F_{x, y}(t+s) \geq \Delta\left(F_{x, z}(t), F_{z, y}(s)\right)$ for all $x, y, z \in X$ and $t, s \geq 0$.

Remark 2.1 In [7], it is pointed out that if $(X, \mathcal{F}, \Delta)$ satisfies the condition $\sup _{0<t<1} \Delta(t, t)=$ 1 , then $(X, \mathcal{F}, \Delta)$ is a Hausdorff topological space in the $(\epsilon, \lambda)$-topology $\mathcal{T}$, i.e., the family of sets $\left\{U_{x}(\epsilon, \lambda): \epsilon>0, \lambda \in(0,1]\right\}(x \in X)$ is a basis of neighborhoods of a point $x$ for $\mathcal{T}$, where $U_{x}(\epsilon, \lambda)=\left\{y \in X: F_{x, y}(\epsilon)>1-\lambda\right\}$.

By virtue of this topology $\mathcal{T}$, a sequence $\left\{x_{n}\right\}$ is said to be $\mathcal{T}$-convergent to $x \in X$ (we write $\left.x_{n} \stackrel{\mathcal{T}}{\rightarrow} x\right)$ if for any given $\epsilon>0$ and $\lambda \in(0,1]$, there exists a positive integer $N=N(\epsilon, \lambda)$ such that $F_{x_{n}, x}(\epsilon)>1-\lambda$ whenever $n \geq N$, which is equivalent to $\lim _{n \rightarrow \infty} F_{x_{n}, x}(t)=1$ for all $t>0 ;\left\{x_{n}\right\}$ is called a $\mathcal{T}$-Cauchy sequence in $(X, \mathcal{F}, \Delta)$ if for any given $\epsilon>0$ and $\lambda \in(0,1]$, there exists a positive integer $N=N(\epsilon, \lambda)$ such that $F_{x_{n}, x_{m}}(\epsilon)>1-\lambda$ whenever $n, m \geq N$; $(X, \mathcal{F}, \Delta)$ is said to be $\mathcal{T}$-complete if each $\mathcal{T}$-Cauchy sequence in $X$ is $\mathcal{T}$-convergent in $X$. Note that in a Menger PM-space, when we write $\lim _{n \rightarrow \infty} x_{n}=x$, it means that $x_{n} \stackrel{\mathcal{T}}{\rightarrow} x$.

Let $(X, \mathcal{F})$ be a PM-space and $A$ be a nonempty subset of $X$. Then the function

$$
D_{A}(t)=\sup _{s<t} \inf _{x, y \in A} F_{x, y}(s), \quad t \in \mathbb{R},
$$

is called the probabilistic diameter of $A$. If $\sup _{t>0} D_{A}(t)=1$, then $A$ is said to be probabilistically bounded.

Let $(X, d)$ be a metric space, $C B(X)$ be the family of all nonempty bounded closed subsets of $X$ and $\delta$ be the Hausdorff metric induced by $d$, that is,

$$
\delta(A, B)=\max \left\{\sup _{x \in A} d(x, B), \sup _{y \in B} d(y, A)\right\}
$$

for any $A, B \in C B(X)$, where $d(x, A)=\inf _{y \in A} d(x, y)$.

Let $(X, \mathcal{F}, \Delta)$ be a Menger space and $\Omega$ be the family of all nonempty probabilistically bounded $\mathcal{T}$-closed subsets of $X$. For any $A, B \in \Omega$, define the distribution functions as follows:

$$
\begin{aligned}
& \tilde{\mathcal{F}}(A, B)(t)=\tilde{F}_{A, B}(t)=\sup _{s<t} \Delta\left(\inf _{x \in A} \sup _{y \in B} F_{x, y}(s), \inf _{y \in B} \sup _{x \in A} F_{x, y}(s)\right), \quad s, t \in \mathbb{R}, \\
& \mathcal{F}(x, A)(t)=F_{x, A}(t)=\sup _{s<t} \sup _{y \in A} F_{x, y}(s), \quad s, t \in \mathbb{R},
\end{aligned}
$$

where $\tilde{\mathcal{F}}$ is called the Menger-Hausdorff metric induced by $\mathcal{F}$.

\section{Remark 2.2 [7]}

(1) $(C B(X), \delta)$ is a metric space. If $(X, d)$ is complete, then $(C B(X), \delta)$ is complete.

(2) Let $(X, d)$ be a metric space. Define a mapping $\mathcal{F}: X \times X \rightarrow \mathscr{D}$ by

$$
\mathcal{F}(x, y)(t)=F_{x, y}(t)=H(t-d(x, y)), \quad \forall x, y \in X, t \in \mathbb{R} .
$$

Then $\left(X, \mathcal{F}, \Delta_{\text {min }}\right)$ is a Menger PM-space induced by $(X, d)$ with $\Delta_{\min }(a, b)=\min \{a, b\}, \forall a, b \in[0,1]$. If $(X, d)$ is complete, then $\left(X, \mathcal{F}, \Delta_{\min }\right)$ is $\mathcal{T}$-complete. 
(3) If we define $\tilde{\mathcal{F}}: C B(X) \times C B(X) \rightarrow \mathscr{D}$ as follows:

$$
\tilde{\mathcal{F}}(A, B)(t)=\tilde{F}_{A, B}(t)=H(t-\delta(A, B)), \quad \forall A, B \in C B(X), t \in \mathbb{R},
$$

then $\tilde{\mathcal{F}}$ is the Menger-Hausdorff metric induced by $\mathcal{F}$. Moreover, if $(X, \mathcal{F}, \Delta)$ is a $\mathcal{T}$-complete Menger PM-space with the $t$-norm $\Delta \geq \Delta_{m}$, where $\Delta_{m}(a, b)=\max \{a+b-$ $1,0\}, \forall a, b \in[0,1]$, then $(\Omega, \tilde{\mathcal{F}}, \Delta)$ is also a $\mathcal{T}$-complete Menger PM-space.

The following lemmas play an important role in proving our main results in Section 3.

Lemma 2.1 [7] Let $(X, \mathcal{F}, \Delta)$ be a Menger PM-space. Then for any $A, B, C \in \Omega$ and any $x, y \in X$, we have the following:

(i) $\tilde{F}_{A, B}(t)=1$ if and only if $A=B$;

(ii) $F_{x, A}(t)=1$ if and only if $x \in A$;

(iii) For any $x \in A, F_{x, B}(t) \geq \tilde{F}_{A, B}(t)$ for all $t \geq 0$;

(iv) $F_{x, A}\left(t_{1}+t_{2}\right) \geq \Delta\left(F_{x, y}\left(t_{1}\right), F_{y, A}\left(t_{2}\right)\right)$ for all $t_{1}, t_{2} \geq 0$;

(v) $F_{x, A}\left(t_{1}+t_{2}\right) \geq \Delta\left(F_{x, B}\left(t_{1}\right), F_{A, B}\left(t_{2}\right)\right)$ for all $t_{1}, t_{2} \geq 0$;

(vi) $\tilde{F}_{A, C}\left(t_{1}+t_{2}\right) \geq \Delta\left(\tilde{F}_{A, B}\left(t_{1}\right), \tilde{F}_{B, C}\left(t_{2}\right)\right)$ for all $t_{1}, t_{2} \geq 0$.

Lemma 2.2 [7] Let $(X, \mathcal{F}, \Delta)$ be a Menger PM-space with a continuous $t$-norm $\Delta$ on $[0,1] \times[0,1], x, y \in X,\left\{x_{n}\right\},\left\{y_{n}\right\} \subset X$ and $x_{n} \stackrel{\mathcal{T}}{\rightarrow} x, y_{n} \stackrel{\mathcal{T}}{\rightarrow} y$. Then $\liminf _{n \rightarrow \infty} F_{x_{n}, y_{n}}(t) \geq F_{x, y}(t)$ for all $t>0$. Particularly, if $F_{x, y}(\cdot)$ is continuous at the point $t_{0}$, then $\lim _{n \rightarrow \infty} F_{x_{n}, y_{n}}\left(t_{0}\right)=$ $F_{x, y}\left(t_{0}\right)$.

Imitating the proof of Lemma 2.2 and using Lemma 2.1, we can easily obtain the following two lemmas.

Lemma 2.3 Let $(X, \mathcal{F}, \Delta)$ be a Menger PM-space with a continuous $t$-norm $\Delta$ on $[0,1] \times$ $[0,1]$ and $(\Omega, \tilde{\mathcal{F}}, \Delta)$ be the induced Menger PM-space, $x \in X, P \in \Omega,\left\{x_{n}\right\} \subset X,\left\{P_{n}\right\} \subset \Omega$ and $x_{n} \stackrel{\mathcal{T}}{\rightarrow} x, P_{n} \stackrel{\mathcal{T}}{\rightarrow} P$. Then $\liminf _{n \rightarrow \infty} F_{x_{n}, P_{n}}(t) \geq F_{x, P}(t)$ for all $t>0$. Particularly, if $F_{x, P}(\cdot)$ is continuous at the point $t_{0}$, then $\lim _{n \rightarrow \infty} F_{x_{n}, P_{n}}\left(t_{0}\right)=F_{x, P}\left(t_{0}\right)$.

Lemma 2.4 Let $(X, \mathcal{F}, \Delta)$ be a Menger PM-space with a continuous $t$-norm $\Delta$ on $[0,1] \times$ $[0,1]$ and $(\Omega, \tilde{\mathcal{F}}, \Delta)$ be the induced Menger PM-space, $P, Q \in \Omega,\left\{P_{n}\right\},\left\{Q_{n}\right\} \subset \Omega$ and $P_{n} \stackrel{\mathcal{T}}{\rightarrow}$ $P, Q_{n} \stackrel{\mathcal{T}}{\rightarrow} Q$. Then $\liminf _{n \rightarrow \infty} \tilde{F}_{P_{n}, Q_{n}}(t) \geq \tilde{F}_{P, Q}(t)$ for all $t>0$. Particularly, if $\tilde{F}_{P, Q}(\cdot)$ is continuous at the point $t_{0}$, then $\lim _{n \rightarrow \infty} \tilde{F}_{P_{n}, Q_{n}}\left(t_{0}\right)=\tilde{F}_{P, Q}\left(t_{0}\right)$.

We recall the definition of compatibility in a hybrid case and weakly compatibility in both single-valued and hybrid case in Menger PM-spaces.

Definition 2.2 [15] Let $(X, \mathcal{F}, \Delta)$ be a Menger PM-space and $(\Omega, \tilde{\mathcal{F}}, \Delta)$ be the induced Menger PM-space. Then $f: X \rightarrow X$ and $F: X \rightarrow \Omega$ are said to be compatible if $f F x \in \Omega$ for all $x \in X$ and $\lim _{n \rightarrow \infty} \tilde{F}_{f F x_{n}, F f x_{n}}(t)=1$ for all $t>0$ whenever $\left\{x_{n}\right\}$ is a sequence in $X$ such that $\lim _{n \rightarrow \infty} f x_{n}=a \in A$ and $\lim _{n \rightarrow \infty} F x_{n}=A \in \Omega$.

Definition $2.3[13]$ Let $(X, \mathcal{F}, \Delta)$ be a Menger PM-space. Then $f: X \rightarrow X$ and $F: X \rightarrow X$ are said to be weakly compatible if they commute at their coincidence points, i.e., $f F x=F f x$ whenever $f x=F x$. 
Definition 2.4 [15] Let $(X, \mathcal{F}, \Delta)$ be a Menger PM-space, $(\Omega, \tilde{\mathcal{F}}, \Delta)$ be the induced Menger PM-space. Then $f: X \rightarrow X$ and $F: X \rightarrow \Omega$ are said to be weakly compatible if they commute at their coincidence points, i.e., $f F x=F f x$ whenever $f x \in F x$.

In the sequel, we will denote by $C(f, F)$ the set of all coincidence points of $f$ and $F$.

We first give the definition of the property (E.A) for a hybrid pair of mappings in Menger PM-spaces.

Definition 2.5 Let $(X, \mathcal{F}, \Delta)$ be a Menger PM-space, $(\Omega, \tilde{\mathcal{F}}, \Delta)$ be the induced Menger PM-space, $f: X \rightarrow X$ be a self-mapping and $F: X \rightarrow \Omega$ be a multivalued mapping. A pair of mappings $(f, F)$ is said to satisfy the property $(E . A)$ if there exists a sequence $\left\{x_{n}\right\}$ in $\mathrm{X}$ and some $a \in X$ and $A \in \Omega$ such that $\lim _{n \rightarrow \infty} f x_{n}=a \in A=\lim _{n \rightarrow \infty} F x_{n}$.

Remark 2.3 Similar to the arguments in [29], by this definition, we can also see that in a hybrid case, any noncompatible mappings satisfy the property (E.A). But the following example shows that the converse is not true.

Example 2.1 Let $X=[0,+\infty)$. Define $\mathcal{F}: X \times X \rightarrow \mathcal{D}$ and $\tilde{\mathcal{F}}: C B(X) \times C B(X) \rightarrow \mathscr{D}$ as follows:

$$
\begin{aligned}
& \mathcal{F}(x, y)(t)=F_{x, y}(t)=H(t-d(x, y)), \quad \forall x, y \in X, t \in \mathbb{R}, \\
& \tilde{\mathcal{F}}(A, B)(t)=\tilde{F}_{A, B}(t)=H(t-\delta(A, B)), \quad \forall A, B \in C B(X), t \in \mathbb{R} .
\end{aligned}
$$

Then by Remark 2.2, we know that $\left(X, \mathcal{F}, \Delta_{\min }\right)$ and $\left(\Omega, \tilde{\mathcal{F}}, \Delta_{\min }\right)$ are both Menger PMspaces. Define $f: X \rightarrow X$ and $F: X \rightarrow \Omega$ as $f x=3 x, F x=[0,1+2 x]$ and take $x_{n}=\frac{1}{n}$. Then $f x_{n} \stackrel{\mathcal{T}}{\rightarrow} 0, F x_{n} \stackrel{\mathcal{T}}{\rightarrow}[0,1]$, which implies that $\lim _{n \rightarrow \infty} f x_{n}=0 \in[0,1]=\lim _{n \rightarrow \infty} F x_{n}$, and so $(f, F)$ satisfies the property (E.A). On the other hand, suppose that $\left\{x_{n}\right\}$ is an arbitrary sequence in $X$ satisfying $\lim _{n \rightarrow \infty} f x_{n}=a \in A=\lim _{n \rightarrow \infty} F x_{n}$ for some $a \in X$ and $A \in \Omega$. Then $f F x_{n}=F f x_{n}=\left[0,1+2 x_{n}\right]$, which implies that $\lim _{n \rightarrow \infty} \tilde{F}_{f F x_{n}, F f x_{n}}(t)=\tilde{F}_{\left[0,1+2 x_{n}\right],\left[0,1+2 x_{n}\right]}(t)=1$ for all $t>0$. So, $f$ and $F$ are compatible mappings.

We now give the definition of the common property (E.A) for two hybrid pairs of mappings in Menger PM-spaces.

Definition 2.6 Let $(X, \mathcal{F}, \Delta)$ be a Menger PM-space and $(\Omega, \tilde{\mathcal{F}}, \Delta)$ be the induced Menger PM-space, $f, g: X \rightarrow X$ and $F, G: X \rightarrow \Omega$. Two pairs of mappings $(f, F)$ and $(g, G)$ are said to satisfy the common property (E.A) if there exist two sequences $\left\{x_{n}\right\},\left\{y_{n}\right\}$ in $X$ and some $u \in X$ and $A, B \in \Omega$ such that

$$
\lim _{n \rightarrow \infty} F x_{n}=A, \quad \lim _{n \rightarrow \infty} G y_{n}=B, \quad \lim _{n \rightarrow \infty} f x_{n}=\lim _{n \rightarrow \infty} g y_{n}=u \in A \cap B .
$$

Example 2.2 Let $(X, d)$ be a metric space, $X=[1,+\infty),(X, \mathcal{F}, \Delta)$ and $(\Omega, \tilde{\mathcal{F}}, \Delta)$ be two Menger PM-spaces induced by $(X, d)$ and $(C B(X), \delta)$, respectively (as in Remark 2.2). Define $f, g: X \rightarrow X$ and $F, G: X \rightarrow \Omega$ as follows:

$$
f x=2+\frac{x}{3}, \quad g x=2+\frac{x}{2}, \quad F x=[1,2+x], \quad G x=\left[3,3+\frac{x}{2}\right], \quad \forall x \in X .
$$


Consider the sequence $\left\{x_{n}\right\}=\left\{3+\frac{1}{n}\right\},\left\{y_{n}\right\}=\left\{2+\frac{1}{n}\right\}$ and denote $A=[1,5], B=[3,4]$.

Since $\tilde{F}_{F x_{n}, A}(t)=H\left(t-\delta\left(F x_{n}, A\right)\right)$, while $\delta\left(F x_{n}, A\right)=\frac{1}{n} \rightarrow 0(n \rightarrow \infty)$, we have $\tilde{F}_{F x_{n}, A}(t) \rightarrow 1, \forall t>0$, i.e., $F x_{n} \stackrel{\mathcal{T}}{\rightarrow} A$. Similarly, we have $\tilde{F}_{G y_{n}, B}(t) \rightarrow 1, \forall t>0$, i.e., $G y_{n} \stackrel{\mathcal{T}}{\rightarrow} B$.

On the other hand, since $F_{f x_{n}, 3}(t)=H\left(t-d\left(f x_{n}, 3\right)\right)$, while $d\left(f x_{n}, 3\right)=\frac{1}{3 n} \rightarrow 0(n \rightarrow \infty)$, we have $F_{f x_{n}, 3}(t) \rightarrow 1, \forall t>0$, i.e., $f x_{n} \stackrel{\mathcal{J}}{\rightarrow} 3 \in A \cap B$ as $n \rightarrow \infty$. Similarly, we have $F_{g y_{n}, 3}(t) \rightarrow 1$, $\forall t>0$, i.e., $g y_{n} \stackrel{\mathcal{\tau}}{\rightarrow} 3 \in A \cap B$ as $n \rightarrow \infty$. Thus, the pairs of mappings $(f, F)$ and $(g, G)$ satisfy the common property (E.A).

\section{Main results}

In this section, we will give the main results of this paper. We first present the following common fixed point theorem for two hybrid pairs of mappings in Menger PM-spaces.

Theorem 3.1 Let $(X, \mathcal{F}, \Delta)$ be a Menger PM-space with $\Delta$ a continuous $t$-norm on $[0,1] \times$ $[0,1]$ and $(\Omega, \tilde{\mathcal{F}}, \Delta)$ be the induced Menger PM-space. Suppose that $f, g: X \rightarrow X$ and $F, G$ : $X \rightarrow \Omega$ are mappings satisfying the following conditions:

(i) $(f, F)$ and $(g, G)$ satisfy the common property (E.A);

(ii) $f(X)$ and $g(X)$ are $\mathcal{T}$-closed subsets of $X$;

(iii) For any $x, y \in X$ with $F x \neq$ Gy and some $1 \leq k<2$,

$$
\tilde{F}_{F x, G y}>\min \left\{F_{f x, g y, \frac{2}{k}}\left[F_{f x, F x} \oplus F_{f x, G y}\right], \frac{2}{k}\left[F_{g y, G y} \oplus F_{g y, F x}\right]\right\},
$$

where a $f(t)$ means $f(a t)$. Then $(f, F)$ and $(g, G)$ each has a coincidence point. Moreover, if $f f v=f v$ for $v \in C(f, F)$ and $g g v=g v$ for $v \in C(g, G)$, then $f, g, F$ and $G$ have a common fixed point in $X$.

Proof Since $(f, F)$ and $(g, G)$ satisfy the common property (E.A), there exist $\left\{x_{n}\right\},\left\{y_{n}\right\} \subset X$ and some $u \in X, A, B \in \Omega$ such that

$$
\lim _{n \rightarrow \infty} F x_{n}=A, \quad \lim _{n \rightarrow \infty} G y_{n}=B, \quad \lim _{n \rightarrow \infty} f x_{n}=\lim _{n \rightarrow \infty} g x_{n}=u \in A \cap B .
$$

Since $f(X)$ is $\mathcal{T}$-closed, there exists some $v \in X$ such that $u=f v$. We claim that $f v \in F v$. Suppose this is not true, that is, $f v \notin F v$. Then from $u=f v \in B$, we have $B \neq F v$. Thus, there exists some $t_{0}>0$ such that

$$
\tilde{F}_{F v, B}\left(\frac{2 t_{0}}{k}\right)>\tilde{F}_{F v, B}\left(t_{0}\right)
$$

(Otherwise, $\forall t>0, F_{F v, B}(t)=F_{F v, B}\left(\frac{2 t}{k}\right)=\cdots=F_{F v, B}\left(\left(\frac{2}{k}\right)^{n} t\right) \rightarrow 1$ as $n \rightarrow \infty$, that is, $F_{F v, B}(t)=$ $1, \forall t>0$, which is a contradiction.)

Without loss of generality, we can assume that $t_{0}$ is a continuous point of $\tilde{F}_{F v, B}(\cdot)$. In fact, by the left continuity of the distribution function, we know that there exists some $\delta>0$ such that

$$
\tilde{F}_{F v, B}\left(\frac{2 t}{k}\right)>\tilde{F}_{F v, B}(t), \quad \forall t \in\left(t_{0}-\delta, t_{0}\right] .
$$

Since the distribution function is nondecreasing, the discontinuous points are at most a countable set. Thus, when $t_{0}$ is not a continuous point of $\tilde{F}_{F v, B}$, we can always choose a point $t_{1}$ in $\left(t_{0}-\delta, t_{0}\right]$ to replace $t_{0}$. 
Noting that $\lim _{n \rightarrow \infty} f x_{n}=u \notin F v$ and $u \in B=\lim _{n \rightarrow \infty} G y_{n}$, we have $F v \neq \lim _{n \rightarrow \infty} G y_{n}$, so there exists some $n_{0} \in N$ such that for all $n \geq n_{0}, G y_{n} \neq F v$.

From (3.1) we know that

$$
\begin{aligned}
& \tilde{F}_{F v, G y_{n}}\left(t_{0}\right) \\
& \quad \geq \min \left\{F_{f v, g y_{n}}\left(t_{0}\right),\left[F_{f v, F v} \oplus F_{f v, G y_{n}}\right]\left(\frac{2}{k} t_{0}\right),\left[F_{g y_{n}, G y_{n}} \oplus F_{g y_{n}, F v}\right]\left(\frac{2}{k} t_{0}\right)\right\} .
\end{aligned}
$$

It is easy to verify that

$$
\liminf _{n \rightarrow \infty}\left[F_{f v, F v} \oplus F_{f v, G y_{n}}\right]\left(\frac{2}{k} t_{0}\right) \geq F_{f v, F v}\left(\frac{2}{k} t_{0}\right)
$$

In fact, for any $\delta \in\left(0, \frac{2}{k} t_{0}\right)$, we have

$$
\left[F_{f v, F v} \oplus F_{f v, G_{y_{n}}}\right]\left(\frac{2}{k} t_{0}\right) \geq \min \left\{F_{f v, F v}\left(\frac{2}{k} t_{0}-\delta\right), F_{f v, G_{y_{n}}}(\delta)\right\} .
$$

Since $f v=u \in B=\lim _{n \rightarrow \infty} G y_{n}$, by Lemma 2.3 and Lemma 2.1(ii), we get

$$
\liminf _{n \rightarrow \infty}\left[F_{f v, F v} \oplus F_{f v, G y_{n}}\right]\left(\frac{2}{k} t_{0}\right) \geq F_{f v, F v}\left(\frac{2}{k} t_{0}-\delta\right) .
$$

Letting $\delta \rightarrow 0$, by the left continuity of the distribution function, we obtain (3.5). Similarly, we can prove that

$$
\liminf _{n \rightarrow \infty}\left[F_{g y_{n}, G y_{n}} \oplus F_{g y_{n}, F_{v}}\right]\left(\frac{2}{k} t_{0}\right) \geq F_{f v, F v}\left(\frac{2}{k} t_{0}\right) .
$$

Noting that $t_{0}$ is the continuous point of $\tilde{F}_{F v, B}(\cdot)$, by Lemma 2.4, we have

$$
\lim _{n \rightarrow \infty} \tilde{F}_{F v, G_{y_{n}}}\left(t_{0}\right)=\tilde{F}_{F v, B}\left(t_{0}\right) .
$$

Thus, letting $n \rightarrow \infty$ in (3.4) and using (3.5) and (3.6), we obtain

$$
\tilde{F}_{F v, B}\left(t_{0}\right) \geq \min \left\{1, F_{f v, F v}\left(\frac{2}{k} t_{0}\right), F_{f v, F v}\left(\frac{2}{k} t_{0}\right)\right\}=F_{f v, F v}\left(\frac{2}{k} t_{0}\right),
$$

that is,

$$
\tilde{F}_{F v, B}\left(t_{0}\right) \geq F_{f v, F v}\left(\frac{2}{k} t_{0}\right) .
$$

But since $f v \in B$, by Lemma 2.1(iii), (3.3) implies that

$$
F_{f v, F v}\left(\frac{2 t_{0}}{k}\right)>\tilde{F}_{F v, B}\left(t_{0}\right)
$$

which is a contradiction. So, we get $f v \in F v$. 
On the other hand, since $g(X)$ is $\mathcal{T}$-closed, there exists some $w \in X$ such that $u=g w$. We claim that $g w \in G w$. Suppose this is not true, that is, $g w \notin G w$. Noting that $u=g w \in A$, we have $A \neq G w$. Similarly, we know that there exists some $s_{0}>0$ such that

$$
\tilde{F}_{A, G w}\left(\frac{2 s_{0}}{k}\right)>\tilde{F}_{A, G w}\left(s_{0}\right) .
$$

Similarly, without loss of generality, we can assume that $s_{0}$ is a continuous point of $\tilde{F}_{A, G w}(\cdot)$.

Noting that $\lim _{n \rightarrow \infty} g y_{n}=u \notin G w$ and $u \in A=\lim _{n \rightarrow \infty} F x_{n}$, we have $\lim _{n \rightarrow \infty} F x_{n} \neq G w$, so there exists some $n_{0} \in N$ such that for all $n \geq n_{0}, F x_{n} \neq G w$.

From (3.1) we know that

$$
\begin{aligned}
& \tilde{F}_{F x_{n}, G w}\left(s_{0}\right) \\
& \quad \geq \min \left\{F_{f x_{n}, g w}\left(s_{0}\right),\left[F_{f x_{n}, F x_{n}} \oplus F_{f x_{n}, G w}\right]\left(\frac{2}{k} s_{0}\right),\left[F_{g w, G w} \oplus F_{g w, F x_{n}}\right]\left(\frac{2}{k} s_{0}\right)\right\} .
\end{aligned}
$$

It is easy to verify that

$$
\liminf _{n \rightarrow \infty}\left[F_{g w, G w} \oplus F_{g w, F x_{n}}\right]\left(\frac{2}{k} s_{0}\right) \geq F_{g w, G w}\left(\frac{2}{k} s_{0}\right) .
$$

In fact, for any $\delta \in\left(0, \frac{2}{k} s_{0}\right)$, we have

$$
\left[F_{g w, G w} \oplus F_{g w, F x_{n}}\right]\left(\frac{2}{k} s_{0}\right) \geq \min \left\{F_{g w, G w}\left(\frac{2}{k} s_{0}-\delta\right), F_{g w, F x_{n}}(\delta)\right\} .
$$

Since $g w=u \in A=\lim _{n \rightarrow \infty} F x_{n}$, by Lemma 2.3 and Lemma 2.1(ii), we get

$$
\liminf _{n \rightarrow \infty}\left[F_{g w, G w} \oplus F_{g w, F x_{n}}\right]\left(\frac{2}{k} s_{0}\right) \geq F_{g w, G w}\left(\frac{2}{k} s_{0}-\delta\right) .
$$

Letting $\delta \rightarrow 0$, by the left continuity of the distribution function, we obtain (3.9). Similarly, we can prove that

$$
\liminf _{n \rightarrow \infty}\left[F_{f x_{n}, F x_{n}} \oplus F_{f x_{n}, G w}\right]\left(\frac{2}{k} s_{0}\right) \geq F_{g w, G w}\left(\frac{2}{k} s_{0}\right) .
$$

Noting that $s_{0}$ is the continuous point of $\tilde{F}_{A, G w}(\cdot)$, by Lemma 2.4 , we have

$$
\lim _{n \rightarrow \infty} \tilde{F}_{F x_{n}, G w}\left(s_{0}\right)=\tilde{F}_{A, G w}\left(s_{0}\right) .
$$

Thus, letting $n \rightarrow \infty$ in (3.8) and using (3.9) and (3.10), we obtain

$$
\tilde{F}_{A, G w}\left(s_{0}\right) \geq \min \left\{1, F_{g w, G w}\left(\frac{2}{k} s_{0}\right), F_{g w, G w}\left(\frac{2}{k} s_{0}\right)\right\}=F_{g w, G w}\left(\frac{2}{k} s_{0}\right),
$$

that is

$$
\tilde{F}_{A, G w}\left(s_{0}\right) \geq F_{g w, G w}\left(\frac{2}{k} s_{0}\right) .
$$


But since $g w \in A$, by Lemma 2.1(iii), (3.7) implies that

$$
F_{g w, G w}\left(\frac{2 s_{0}}{k}\right)>\tilde{F}_{A, G w}\left(s_{0}\right)
$$

which is a contradiction. So, we get $g w \in G w$. Therefore, we have proved $u=f v \in F v$, and $u=g w \in G w$, i.e., $v$ is a coincidence point of $(f, F)$ and $w$ is a coincidence point of $(g, G)$.

Since $v \in C(f, F)$ and $w \in C(g, G)$, we have $u=f v=f f v=f u \in F v$ and $u=g w=g g w=g u \in$ $G w$. Next, we prove that $F v=F u$ and $G w=G u$.

First, we assert that $F v=G w$. In fact, suppose that $F v \neq G w$. Then by (3.1), there exists some $t_{1}>0$ such that

$$
\tilde{F}_{F v, G w}\left(t_{1}\right)>\min \left\{F_{f v, g w}\left(t_{1}\right),\left[F_{f v, F v} \oplus F_{f v, G w}\right]\left(\frac{2}{k} t_{1}\right),\left[F_{g w, G w} \oplus F_{g w, F v}\right]\left(\frac{2}{k} t_{1}\right)\right\} .
$$

This implies that

$$
1=F_{u, G w}\left(t_{1}\right) \geq \tilde{F}_{F v, G w}\left(t_{1}\right)>1,
$$

which is a contradiction, and thus we have $F v=G w$.

Similarly, we can prove that $F u=G w$. In fact, suppose that $F u \neq G w$. Then by (3.1), there exists some $t_{2}>0$ such that

$$
\tilde{F}_{F u, G w}\left(t_{2}\right)>\min \left\{F_{f u, g w}\left(t_{2}\right),\left[F_{f u, F u} \oplus F_{f u, G w}\right]\left(\frac{2}{k} t_{2}\right),\left[F_{g w, G w} \oplus F_{g w, F u}\right]\left(\frac{2}{k} t_{2}\right)\right\}
$$

This implies that

$$
F_{u, F u}\left(t_{2}\right) \geq \tilde{F}_{F u, G w}\left(t_{2}\right)>F_{u, F u}\left(\frac{2}{k} t_{2}\right)
$$

which is a contradiction, and thus we have $F u=G w$. Combining these two facts yields $F v=F u$.

Next, we assert that $F v=G u$. Suppose that $F v \neq G u$. Again by (3.1), there exists some $t_{3}>0$ such that

$$
\tilde{F}_{F v, G u}\left(t_{3}\right)>\min \left\{F_{f v, g u}\left(t_{3}\right),\left[F_{f v, F v} \oplus F_{f v, G u}\right]\left(\frac{2}{k} t_{3}\right),\left[F_{g u, G u} \oplus F_{g u, F v}\right]\left(\frac{2}{k} t_{3}\right)\right\} .
$$

This implies that

$$
F_{u, G u}\left(t_{3}\right) \geq \tilde{F}_{F v, G u}\left(t_{3}\right)>F_{u, G u}\left(\frac{2}{k} t_{3}\right),
$$

which is a contradiction, and so we have $F v=G u$. Combining this with $F v=G w$, we obtain $G w=G u$.

Thus, we have $u=f u \in F u$ and $u=g u \in G u$, that is, $u$ is the common fixed point of $f, g$, $F$ and $G$. This completes the proof.

From the proof of Theorem 3.1, we can similarly prove the following result. 
Theorem 3.2 Let $(X, \mathcal{F}, \Delta)$ be a Menger PM-space with $\Delta$ a continuous $t$-norm on $[0,1] \times[0,1]$ and let $(\Omega, \tilde{\mathcal{F}}, \Delta)$ be the induced Menger PM-space. Suppose that $f, g: X \rightarrow X$ and $F, G: X \rightarrow \Omega$ are mappings satisfying the conditions (i)-(ii) of Theorem 3.1 and the following:

(iii)' For any $x, y \in X$ with $F x \neq$ Gy and some $1 \leq k<2$,

$$
\tilde{F}_{F x, G y}>\min \left\{F_{f x, g y}, \frac{2}{k}\left[F_{f x, F x} \oplus F_{g y, G y}\right],{ }_{2}\left[F_{f x, G y} \oplus F_{g y, F x}\right]\right\}
$$

where af $(t)$ means $f(a t)$. Then $(f, F)$ and $(g, G)$ each has a coincidence point. Moreover, if $f f v=f v$ for $v \in C(f, F)$ and $g g v=g v$ for $v \in C(g, G)$, then $f, g, F$ and $G$ have a common fixed point in $X$.

Setting $f=g$ in Theorem 3.1, we obtain the following corollary.

Corollary 3.1 Let $(X, \mathcal{F}, \Delta)$ be a Menger space with $\Delta$ a continuous t-norm on $[0,1] \times[0,1]$ and let $(\Omega, \tilde{\mathcal{F}}, \Delta)$ be the induced Menger space. Suppose that $f: X \rightarrow X$ and $F, G: X \rightarrow \Omega$ are mappings satisfying the following conditions:

(i) $(f, F)$ and $(f, G)$ satisfy the common property $(E . A)$;

(ii) $f(X)$ is a $\mathcal{T}$-closed subset of $X$;

(iii) For any $x, y \in X$ with $F x \neq$ Gy and some $1 \leq k<2$,

$$
\tilde{F}_{F x, G y}>\min \left\{F_{f x, f y}, \frac{2}{k}\left[F_{f x, F x} \oplus F_{f x, G y}\right], \frac{2}{k}\left[F_{f y, G y} \oplus F_{f y, F x}\right]\right\},
$$

where $f(t)$ means $f(a t)$. Then $f, F$ and $G$ have a coincidence point. Moreover, ifff $v=f v$ for $v \in C(f, F)$ and $v \in C(f, G)$, then $f, F$ and $G$ have a common fixed point in $X$.

Setting $f=g$ and $F=G$, we have the following corollary.

Corollary 3.2 Let $(X, \mathcal{F}, \Delta)$ be a Menger space with $\Delta$ a continuoust-norm on $[0,1] \times[0,1]$ and let $(\Omega, \tilde{\mathcal{F}}, \Delta)$ be the induced Menger space. Suppose that $f: X \rightarrow X$ and $F: X \rightarrow \Omega$ are mappings satisfying the following conditions:

(i) $(f, F)$ satisfies the property (E.A);

(ii) $f(X)$ is a $\mathcal{T}$-closed subset of $X$;

(iii) For any $x, y \in X$ with $x \neq y$ and some $1 \leq k<2$,

$$
\tilde{F}_{F x, F y}>\min \left\{F_{f x, f y}, \frac{2}{k}\left[F_{f x, F x} \oplus F_{f x, F y}\right], \frac{2}{k}\left[F_{f y, F y} \oplus F_{f y, F x}\right]\right\}
$$

where a $f(t)$ means $f(a t)$. Then $f$ and $F$ have a coincidence point. Moreover, ifffv $=f v$ for $v \in C(f, F)$, then $f$ and $F$ have a common fixed point in $X$.

If $f, g, F$ and $G$ are all single-valued mappings, then we have the following corollary.

Corollary 3.3 Let $(X, \mathcal{F}, \Delta)$ be a Menger space with $\Delta$ a continuous $t$-norm on $[0,1] \times$ $[0,1]$. Suppose that $f, g, F, G: X \rightarrow X$ are self-mappings satisfying the following conditions:

(i) $(f, F)$ and $(g, G)$ satisfy the common property (E.A);

(ii) $f(X)$ and $g(X)$ are $\mathcal{T}$-closed subsets of $X$; 
(iii) For any $x, y \in X$ with $F x \neq$ Gy and some $1 \leq k<2$,

$$
\left.\tilde{F}_{F x, G y}>\min \left\{F_{f x, g y} \frac{2}{k}\left[F_{f x, F x} \oplus F_{f x, G y}\right]\right]_{\frac{2}{k}}\left[F_{g y, G y} \oplus F_{g y, F x}\right]\right\},
$$

where af $f(t)$ means $f(a t)$. Then $(f, F)$ and $(g, G)$ each has a coincidence point. Moreover, if $f f v=f v$ for $v \in C(f, F)$ and $g g v=g v$ for $v \in C(g, G)$, then $f, g, F$ and $G$ have a common fixed point in $X$.

Remark 3.1 We would like to point out here that in the condition (iii), we use 'for any $x, y \in X$ with $F x \neq G y$ ' instead of 'for any $x, y \in X$ with $x \neq y$ ' as in Theorem 2.1 of [30] when we consider two pairs of mappings. Moreover, comparing our Corollary 3.3 with Theorem 2.1 of [30], one can find that we use the condition ' $f f v=f v$ for $v \in C(f, F)$ and $g g v=g v$ for $v \in C(g, G)$ ' instead of weakly compatibility condition for two hybrid pairs. In fact, in a hybrid case, even if $(f, F)$ and $(g, G)$ are weakly compatible, we still cannot obtain the conclusion.

Remark 3.2 Similarly, some other corresponding corollaries can be obtained from Theorem 3.2. For simplicity, we omit them here. Also, it is worth mentioning that in all of the above theorems and corollaries, we do not need any condition on the continuity or the containment of the ranges of involved mappings.

\section{Common fixed point results in metric spaces}

In this section, we use the results in Section 3 to get some corresponding results in metric spaces.

Theorem 4.1 Let $(X, d)$ be a metric space. Suppose that $f, g: X \rightarrow X$ and $F, G: X \rightarrow C B(X)$ are mappings satisfying the following conditions:

(i) $(f, F)$ and $(g, G)$ satisfy the common property $(E . A)$;

(ii) $f(X)$ and $g(X)$ are closed subsets of $X$;

(iii) For any $x, y \in X$ with $F x \neq$ Gy and some $1 \leq k<2$,

$$
\begin{aligned}
& \delta(F x, G y) \\
& \quad<\max \left\{d(f x, g y), \frac{k}{2}[d(f x, F x)+d(f x, G y)], \frac{k}{2}[d(g y, G y)+d(g y, F x)]\right\} .
\end{aligned}
$$

Then $(f, F)$ and $(g, G)$ each has a coincidence point. Moreover, ifffv $=f v$ for $v \in C(f, F)$ and $g g v=g v$ for $v \in C(g, G)$, then $f, g, F$ and $G$ have a common fixed point in $X$.

Proof Let $\left(X, \mathcal{F}, \Delta_{\text {min }}\right)$ be the induced Menger space by $(X, d)$ and $\left(\Omega, \tilde{\mathcal{F}}, \Delta_{\text {min }}\right)$ be the induced Menger space by $(C B(X), \delta)$. Then by Remark 2.2, it is easy to see that Theorem 4.1(i) and (ii) imply Theorem 3.1(i) and (ii). Now we show that Theorem 4.1(iii) implies Theorem 3.1(iii).

We first verify that for any $x, y \in X$ with $F x \neq G y$ and $t>0$, the following holds:

$$
\tilde{F}_{F x, G y}(t) \geq \min \left\{F_{f x, g y}(t),\left[F_{f x, F x} \oplus F_{f x, G y}\right]\left(\frac{2}{k} t\right),\left[F_{g y, G y} \oplus F_{g y, F x}\right]\left(\frac{2}{k} t\right)\right\} .
$$

If $t>\delta(F x, G y)$, then $\tilde{F}_{F x, G y}(t)=1$, and thus (4.2) obviously holds. 
If $t \leq \delta(F x, G y)$, we consider the following three cases:

Case (I): $t<d(f x, g y)$. In this case, $F_{f x, g y}(t)=0$, and thus (4.2) holds.

Case (II): $t<\frac{k}{2}[d(f x, F x)+d(f x, G y)]$, that is, $\frac{2}{k} t<d(f x, F x)+d(f x, G y)$. Then for any $t_{1}, t_{2}>0$ with $t_{1}+t_{2}=\frac{2}{k} t$, we have $d(f x, F x)>t_{1}$ or $d(f x, G y)>t_{2}$, which implies that $F_{f x, F x}\left(t_{1}\right)=0$ or $F_{f x, G y}\left(t_{2}\right)=0$. Hence,

$$
\left[F_{f x, F x} \oplus F_{f x, G y}\right]\left(\frac{2}{k} t\right)=\sup _{t_{1}+t_{2}=\frac{2 t}{k}} \min \left\{F_{f x, F x}\left(t_{1}\right), F_{f x, G y}\left(t_{2}\right)\right\}=0
$$

and so (4.2) holds.

Case (III): $t<\frac{k}{2}[d(g y, G y)+d(g y, F x)]$. Similar to Case (II), we can prove that $\left[F_{g y, G y} \oplus\right.$ $\left.F_{g y, F x}\right]\left(\frac{2}{k} t\right)=0$, so (4.2) holds.

From the above discussions, we conclude that (4.2) is always true.

Next, by (4.1), there exists some $t_{0}>0$ such that

$$
\delta(F x, G y)<t_{0}<\max \left\{d(f x, g y), \frac{k}{2}[d(f x, F x)+d(f x, G y)], \frac{k}{2}[d(g y, G y)+d(g y, F x)]\right\} .
$$

This implies that $\tilde{F}_{F x, G y}\left(t_{0}\right)=1$ and

$$
\min \left\{F_{f x, g y}\left(t_{0}\right),\left[F_{f x, F x} \oplus F_{f x, G y}\right]\left(\frac{2}{k} t_{0}\right),\left[F_{g y, G y} \oplus F_{g y, F x}\right]\left(\frac{2}{k} t_{0}\right)\right\}=0,
$$

which yields that

$$
\tilde{F}_{F x, G y}\left(t_{0}\right)>\min \left\{F_{f x, g y}\left(t_{0}\right),\left[F_{f x, F x} \oplus F_{f x, G y}\right]\left(\frac{2}{k} t_{0}\right),\left[F_{g y, G y} \oplus F_{g y, F x}\right]\left(\frac{2}{k} t_{0}\right)\right\} .
$$

Combining (4.2) with (4.3), we know that (3.1) holds.

Similarly, from Theorem 3.2, we can obtain the following theorem.

Theorem 4.2 Let $(X, d)$ be a metric space. Suppose that $f, g: X \rightarrow X$ and $F, G: X \rightarrow C B(X)$ are mappings satisfying the following conditions:

(i) $(f, F)$ and $(g, G)$ satisfy the common property (E.A);

(ii) $f(X)$ and $g(X)$ are closed subsets of $X$;

(iii) For any $x, y \in X$ with $F x \neq$ Gy and some $1 \leq k<2$,

$$
\begin{aligned}
& \delta(F x, G y) \\
& \quad<\max \left\{d(f x, g y), \frac{k}{2}[d(f x, F x)+d(g y, G y)], \frac{1}{2}[d(f x, G y)+d(g y, F x)]\right\} .
\end{aligned}
$$

Then $(f, F)$ and $(g, G)$ each has a coincidence point. Moreover, ifffv $=f v$ for $v \in C(f, F)$ and $g g v=g \nu$ for $v \in C(g, G)$, then $f, g, F$ and $G$ have a common fixed point in $X$.

Remark 4.1 Note that when $k=1$, then (4.4) becomes (2.3) in Theorem 2.3 of [24]. Similar to Remark 3.1, we should state 'for any $x, y \in X$ with $F x \neq G y$ ' here instead of 'for any $x, y \in X$ with $x \neq y$ ' as in [24]. Moreover, we only need ' $f f v=f v$ for $v \in C(f, F)$ and $g g v=g v$ for 
$v \in C(g, G)$ ' to guarantee the existence of common fixed points of $f, g, F$ and $G$. In fact, the condition ' $f$ is $F$-weakly commuting for $v \in C(f, F)$ and $g$ is $G$-weakly commuting for $v \in C(g, G)$ ' is superfluous in Theorem 2.3 of [24].

Remark 4.2 By Corollary 3.1-Corollary 3.3 and Remark 3.2, we can also obtain some other corresponding corollaries for common fixed point theorems in metric spaces, which are the generalizations of many known results $(e . g .,[22,23])$. For the sake of simplicity, we omit them here.

\section{An application}

In this section, we will provide an example to exemplify the validity of the main result of this paper.

Example 5.1 Consider $X=(-1,1)$ and define $F_{x, y}(t)=\frac{t}{t+|x-y|}$ for all $x, y \in X$ with $t>0$. Also, let $C B(X)$ be the family of nonempty bounded closed subsets of $X$ and define $\tilde{F}_{A, B}(t)=$ $\frac{t}{t+\delta(A, B)}$ for all $A, B \in C B(X)$ with $t>0$. Then $(X, \mathcal{F}, \Delta)$ and $(\Omega, \tilde{\mathcal{F}}, \Delta)$ are both Menger PMspaces with $\Delta(a, b)=\min \{a, b\}$, where $\Omega$ is the family of all nonempty probabilistically bounded $\mathcal{T}$-closed subsets of $X$. Define $f, g: X \rightarrow X$ and $F, G: X \rightarrow \Omega$ as follows:

$$
\begin{aligned}
& f x= \begin{cases}\frac{4}{5}, & \text { if } x \in\left(-1,-\frac{1}{2}\right) \cup\left(\frac{1}{2}, 1\right) ; \\
\frac{x}{2}, & \text { if } x \in\left[-\frac{1}{2}, \frac{1}{2}\right],\end{cases} \\
& g x= \begin{cases}-\frac{4}{5}, & \text { if } x \in\left(-1,-\frac{1}{2}\right) \cup\left(\frac{1}{2}, 1\right) ; \\
-\frac{x}{2}, & \text { if } x \in\left[-\frac{1}{2}, \frac{1}{2}\right],\end{cases} \\
& F x= \begin{cases}{\left[0, \frac{3}{5}\right],} & \text { if } x \in\left(-1,-\frac{1}{2}\right) \cup\left(\frac{1}{2}, 1\right) ; \\
{\left[\frac{x}{4}, 0\right],} & \text { if } x \in\left[-\frac{1}{2}, 0\right] ; \\
{\left[0, \frac{x}{4}\right],} & \text { if } x \in\left[0, \frac{1}{2}\right],\end{cases} \\
& G x= \begin{cases}{\left[-\frac{3}{5}, 0\right],} & \text { if } x \in\left(-1,-\frac{1}{2}\right) \cup\left(\frac{1}{2}, 1\right) ; \\
{\left[0,-\frac{x}{4}\right],} & \text { if } x \in\left[-\frac{1}{2}, 0\right] ; \\
{\left[-\frac{x}{4}, 0\right],} & \text { if } x \in\left[0, \frac{1}{2}\right] .\end{cases}
\end{aligned}
$$

Consider the sequences $\left\{x_{n}=\frac{1}{n+1}\right\}$ and $\left\{y_{n}=\frac{-1}{n+1}\right\}$ in $X$, then

$$
\lim _{n \rightarrow \infty} f x_{n}=\lim _{n \rightarrow \infty} g y_{n}=0 \text { and } \lim _{n \rightarrow \infty} F x_{n}=\lim _{n \rightarrow \infty} G y_{n}=\{0\}
$$

which shows that $(f, F)$ and $(g, G)$ satisfy the common property (E.A). Also, $f(X)$ and $g(X)$ are $\mathcal{T}$-closed subsets of $X$. By a routine calculation, one can verify the inequality (3.1) for any $x, y \in X$ with $F x \neq G y$ and some $1 \leq k<2$.

In fact, if $x \in\left(-1,-\frac{1}{2}\right) \cup\left(\frac{1}{2}, 1\right)$ and $y \in\left[-\frac{1}{2}, 0\right]$, then for $t>0$, we have

$$
\begin{aligned}
\tilde{F}_{F x, G y}(t) & =\frac{t}{t+\frac{3}{5}+\frac{y}{4}}>\frac{t}{t+\frac{4}{5}+\frac{y}{2}}=F_{f x, g y}(t) \\
& \geq \min \left\{F_{f x, g y}(t),_{\frac{2}{k}}\left[F_{f x, F x} \oplus F_{f x, G y}\right](t),_{\frac{2}{k}}\left[F_{g y, G y} \oplus F_{g y, F x}\right](t)\right\} .
\end{aligned}
$$


If $x \in\left(-1,-\frac{1}{2}\right) \cup\left(\frac{1}{2}, 1\right)$ and $y \in\left[0, \frac{1}{2}\right]$, then for $t>0$, we have

$$
\begin{aligned}
\tilde{F}_{F x, G y}(t) & =\frac{t}{t+\frac{3}{5}}>\frac{t}{t+\frac{4}{5}+\frac{y}{2}}=F_{f x, g y}(t) \\
& \geq \min \left\{F_{f x, g y}(t),_{\frac{2}{k}}\left[F_{f x, F x} \oplus F_{f x, G y}\right](t),_{\frac{2}{k}}\left[F_{g y, G y} \oplus F_{g y, F x}\right](t)\right\}
\end{aligned}
$$

Similarly, it is easy to verify (3.1) for other cases. Thus, all the conditions of Theorem 3.1 are satisfied and 0 is the unique coincidence point of $(f, F)$ and $(g, G)$. Furthermore, noting that $f f 0=f 0$ and $g g 0=g 0,0$ remains the common fixed point of $(f, F)$ and $(g, G)$.

It is worth pointing out that many theorems in some corresponding published papers cannot be applied to this example since Theorem 3.1 does not require any condition on the containment of the ranges of involved mappings, and in this example we also do not require such containment.

\section{Competing interests}

The authors declare that they have no competing interests.

\section{Authors' contributions}

All authors contributed equally. All authors read and approved the final manuscript.

\section{Author details}

'Department of Mathematics, Nanchang University, Nanchang, 330031, P.R. China. ${ }^{2}$ School of Computer and Information Engineering, Zhejiang Gongshang University, Hangzhou, 310018, P.R. China.

\section{Acknowledgements}

The authors would like to thank the editor and the referees for their constructive comments and suggestions. The research was supported by the Natural Science Foundation of China (11071108), the Natural Science Foundation of Jiangxi Province of China (2010GZS0147, 20114BAB201003), and partly supported by the Humanities and Social Sciences Foundation of Ministry of Education of China (12YJC630091) and the Natural Science Foundation of Zhejiang Province of China (LQ12G02007).

Received: 25 June 2012 Accepted: 14 January 2013 Published: 6 February 2013

\section{References}

1. Menger, K: Statistical metrics. Proc. Natl. Acad. Sci. USA 28, 535-537 (1942)

2. Schweizer, B, Sklar, A: Statistical metric spaces. Pac. J. Math. 10, 313-334 (1960)

3. Schweizer, B: Commentary on probabilistic geometry. In: Schweizer, B, Sklar, A, Sigmund, K et al. (eds.) Karl Menger, Selecta Mathematica, vol. 2, pp. 409-432. Springer, New York (2003)

4. Schweizer, B, Sklar, A: Probabilistic Metric Spaces. North-Holland, Amsterdam (1983)

5. El Naschie, MS: A review of applications and results of E-infinity theory. Int. J. Nonlinear Sci. Numer. Simul. 8, 11-20 (2007)

6. Hadžić, O, Pap, E: Fixed Point Theory in Probabilistic Metric Spaces. Kluwer Academic, Dordrecht (2001)

7. Chang, SS, Cho, YJ, Kang, SM: Nonlinear Operator Theory in Probabilistic Metric Spaces. Nova Science Publishers, New York (2001)

8. Zhu, CX: Several nonlinear operator problems in the Menger PN space. Nonlinear Anal. 65, 1281-1284 (2006)

9. Zhu, CX: Research on some problems for nonlinear operators. Nonlinear Anal. 71, 4568-4571 (2009)

10. Jungck, G: Compatible mappings and common fixed points. Int. J. Math. Math. Sci. 9, 771-779 (1986)

11. Jungck, G: Common fixed points for non continuous non self maps on non metric spaces. Far East J. Math. Sci. 4, 199-215 (1996)

12. Mishra, SN: Commond fixed points of compatible mappings in PM-spaces. Math. Jpn. 36, 436-440 (1991)

13. Singh, B, Jain, S: A fixed point theorem in Menger space through weak compatibility. J. Math. Anal. Appl. 301, 439-448 (2005)

14. Razani, A, Shirdaryazdi, M: A common fixed point theorem of compatible maps in Menger space. Chaos Solitons Fractals 32, 26-34 (2007)

15. $\mathrm{Wu}, \mathrm{ZQ}, \mathrm{Zhu}, \mathrm{CX}$, Chen, XL: Fixed point theorems and coincidence point theorems for hybrid contractions in PM-spaces. Acta Anal. Funct. Appl. 10(4), 339-345 (2008) (in Chinese)

16. Fang, JX: Common fixed point theorems of compatible and weakly compatible maps in Menger spaces. Nonlinear Anal. 71, 1833-1843 (2009)

17. Pant, RP: Common fixed points of contractive maps. J. Math. Anal. Appl. 226, 251-258 (1998)

18. Pant, RP: R-weakly commutativity and common fixed points. Soochow J. Math. 25, 37-42 (1999)

19. Pant, RP, Pant, V: Common fixed under strict contractive conditions. J. Math. Anal. Appl. 248, 327-332 (2000)

20. Pant, RP, Pant, V, Jha, K: Note on common fixed points under strict contractive conditions. J. Math. Anal. Appl. 274 879-880 (2002) 
21. Aamri, M, El Moutawakil, D: Some new common fixed point theorems under strict contractive conditions. J. Math. Anal. Appl. 270, 181-188 (2002)

22. Kamran, T: Coincidence and fixed points for hybrid strict contractions. J. Math. Anal. Appl. 299, 235-241 (2004)

23. Sintunavarat, W, Kumam, P: Coincidence and common fixed points for hybrid strict contraction without the weakly commuting condition. Appl. Math. Lett. 22, 1877-1881 (2009)

24. Liu, YC, Wu, J, Li, ZX: Common fixed points of single-valued and multivalued maps. Int. J. Math. Math. Sci. 19, 3045-3055 (2005)

25. Imdad, M, Ali, J, Khan, L: Coincidence and fixed points in symmetric spaces under strict contractions. J. Math. Anal. Appl. 320, 352-360 (2006)

26. Imdad, M, Ali, J, Khan, L: Corrigendum to 'Coincidence and fixed points in symmetric spaces under strict contractions' [J. Math. Anal. Appl. 320 (2006) 352-360]. J. Math. Anal. Appl. 329, 752 (2007)

27. Aliouche, A: A common fixed point theorem for weakly compatible mappings in symmetric spaces satisfying a contractive condition of integral type. J. Math. Anal. Appl. 322, 796-802 (2006)

28. Djoudi, A, Aliouche, A: Common fixed point theorems of Gregus type for weakly compatible mappings satisfying contractive conditions of integral type. J. Math. Anal. Appl. 329, 31-45 (2007)

29. Fang, JX, Gang, Y: Common fixed point theorems under strict contractive conditions in Menger spaces. Nonlinear Anal. 70, 184-193 (2009)

30. Ali, J, Imdad, M, Mihet, D, Tanveer, M: Common fixed points of strict contractions in Menger spaces. Acta Math. Hung. 132(4), 367-386 (2011)

doi:10.1186/1687-1812-2013-25

Cite this article as: Wu et al.: Common fixed point theorems for two hybrid pairs of mappings satisfying the common property (E.A) in Menger PM-spaces. Fixed Point Theory and Applications 2013 2013:25.

\section{Submit your manuscript to a SpringerOpen ${ }^{\circ}$ journal and benefit from:}

- Convenient online submission

- Rigorous peer review

- Immediate publication on acceptance

Open access: articles freely available online

High visibility within the field

- Retaining the copyright to your article 